\title{
Performance Analysis of Image Fusion Techniques for Sonar Image Enhancement
}

\author{
J. Alavandan \\ Associate Professor \& Head, Dept of Computer \\ Science \& Applications, Jawahar Science College, \\ Neyveli, Tamilnadu, India
}

\author{
S.Santhosh Baboo \\ Reader, Postgraduate \& Research Department of \\ Computer Science, \\ Dwaraka Doss Goverdhan Doss Vaishnav \\ College, \\ Chennai, India
}

\begin{abstract}
SONAR (Sound Navigation and Ranging) is a technology that is used to observe Earth surfaces with focus on underwater applications like sea-bed imaging, depth sounding and fishecholocation. The captured sonar images are often disturbed by various factors like the transmission of limited range of light, disturbance of lightening, low contrast and blurring of image, color diminishing during capturing and noise. These disturbances affect image quality which often lead to incorrect analysis and has to be handled carefully. To efficiently analyze an image, the quality of the image should be high standard and thus, enhancement of image quality has become imperative in image analysis systems. In this paper, two techniques, Laplacian Pyramid-based image fusion and Wavelet-based image fusion algorithms are considered and their applicability to fuse sonar images to construct an enhanced image is analyzed. The paper considers various pictures from single sensor and performance evaluation was performed in terms of Peak Signal to Noise Ratio, Figure of Merit and Speed of algorithms. Experiments showed that wavelets produced fast and better quality images, while edges were better preserved by pixel-based algorithms.
\end{abstract}

\section{Keywords}

Image Fusion, Laplacian-Pyramid, Pixel-based, Sonar Image Enhancement, Wavelet-based.

\section{INTRODUCTION}

SONAR (Sound Navigation and Ranging), is a technology that is used to observe Earth surfaces with focus on underwater applications like sea-bed imaging, depth sounding and fish-echolocation. Sonar acoustic cameras capture oceanic images in two-dimensional format and are used by sonar imaging systems during the study of water surface of the Earth. As underwater environments are dynamic and complex, obtaining a clear picture of the obstacles and movements of objects in this environment is critical and challenging. The captured Sonar images often are disturbed by various factors like the transmission of limited range of light, disturbance of lightening, low contrast and blurring of image, color diminishing during capturing and noise. These disturbances affect image quality which often lead to incorrect analysis and has to be handled carefully. To efficiently analyze an image, the quality of the image should be high standard and thus, enhancement of image quality has become imperative in image analysis systems. Image enhancement techniques like histogram equalization, image smoothening, image sharpening, contrast adjustment, edge or boundary enhancement and denoising are generally used for this purpose. In this paper, another type of enhancement using image fusion is presented.

Image fusion is an effective way for optimum utilization of large volumes of image from multiple sources. Multiple image fusion seeks to combine information from multiple sources to achieve inferences that are not feasible from a single source. It is a process of combining relevant information from a set of images, into a single image, wherein the resultant fused image will be more informative and complete than any of the input images (Elaksher, 2008) [1].

In oceanography, image fusion provides an effective way of reducing the increasing volume of information while at the same time extracting all the useful information from the source images. Image fusion as a solution to enhance sonar images is beneficial in many situations including the following. First, multi-sensor data often presents complementary information about the region being surveyed. Second, images with different characteristics and different resolutions obtained at different time may provide complementary information about the surface. Almost all existing surface capturing devices, provide images at both higher and lower spatial resolutions. Irrespective of the resolution and characteristics, the images are often degraded by the presence of noise. In all these situations, image fusion technology provides an effective method to enable comparison and analysis of such data and produce a new integrated image while retaining the important feature from these images which vary in terms of characteristics, degradation and resolution.

Various methods exist for fusing images. They include pixellevel fusion, feature-level fusion and decision-level fusion. Pixel-based fusion is performed on a pixel-by-pixel basis. It generates a fused image in which information associated with each pixel is determined from a set of pixels in source images to improve the performance of image processing tasks such as segmentation. Feature-based fusion at feature level requires an extraction of objects recognized in the various data sources. It requires the extraction of salient features which are depending on their environment such as pixel intensities, edges or textures. These similar features from input images are fused. This category consists of merging information at a higher level of abstraction, combines the results from multiple algorithms to yield a final fused decision. Input images are processed individually for information extraction. The 
obtained information is then combined applying decision rules to reinforce common interpretation.

This paper is focused on comparing the performance of the two well-known techniques, pixel-level and feature-based fusion algorithms, to improve the quality of the sonar images. The pixel-level technique considered is the Laplacian pyramid image fusion algorithm. For feature-based techniques, wavelets are considered. These techniques were selected because of their popularity and efficiency in improving the quality of images. The rest of the paper is organized in the following manner. Section 2 presents a brief review of various works related to the two selected techniques. Section 3 describes the methodology behind the two selected techniques. Section 4 presents the comparative results, while Section 5 concludes the work with future research directions.

\section{LITERATURE STUDY}

All materials According to Keys et al. (1990) [2] fusing image data leads to a more accurate and enhanced data which increases the reliability of an image and increased utility. Fused data paves way for robust operational performance, i.e., increased confidence, reduced ambiguity, improved reliability and improved classification. Studying the literature, image fusion is applied to digital imagery in order to (i) sharpen images (ii) Enhance certain features not visible in either of the single data alone (iii) Complement data sets for improved classification (iv) Substitute missing information in one image with signals from another sensor image. This process is also called filling gaps. The following sections describe studies that focus on each of the above listed applications.

\subsection{Image sharpening}

Image fusion can be used as a tool to increase the spatial resolution. In that case high-resolution panchromatic imagery is fused with low-resolution often multi-spectral image data. A distinction has to be made between the pure visual enhancement (superimposition) and real interpolation of data to achieve higher resolution (wavelets). Wavelets were initially exploited for image fusion by Mangolini (1994) [3] and Ranchin et al. (1996) [4]. Here, the spectral resolution is preserved while a higher spatial resolution is incorporated which represents the information content of the images in much more detail (Pellemans et al., 1993 [5]). Examples of fusing images for resolution enhancement were studied by Simard (2002) [6], simulated data (Price (2007) [7]); Ranchin et al. (1996) [4].

\subsection{Feature enhancement}

Taking advantage of the different physical nature of microwave and optical sensor systems the fusion of those data results in an enhancement of various features observed (Yesou et al., 1993b [8]). The feature enhancement capability of image fusion is visually apparent in situations where the input set of images is superior to the original data (Keys et al., 1990 [2]). Multisensor image fusion enhances semantic capabilities of the images and yields information which is otherwise unavailable or hard to obtain from single sensor data (Mitiche and Aggarwal, 2006 [9]). Further, feature enhancement facilitates extracting useful information or objects from the fused images (Welch and Ehlers, 2008 [10]).

\subsection{Improved classification}

Fusion of images also improves the image classification accuracy. The microwave and optical sensors images offer complementary information that helps in discriminating the different classes. The use of multisensor data in image classification becomes more and more popular with the increased availability of sophisticated software and hardware facilities to handle the increasing volumes of data. A new trend in this respect is the Artificial Neural Network (ANN) approach since the conventional statistical techniques were found inappropriate for processing multisensor data.

\subsection{Overcoming gaps}

The satellite remote sensors images are mostly influenced by a number of effects based on the carrier frequency of the electromagnetic waves. It is well known that VIR sensors are hindered by clouds to obtain information on the observed objects on Earth. Even the shadows of the clouds influence the interpretability of the imagery. SAR on the other hand suffers from severe terrain induced geometric distortions based on its side-looking geometry (layover, foreshortening and shadow). To overcome these influences it is possible to combine different images acquired by the same or a different devices or intruments. Apart from creating simple or complex mosaics there are other techniques such as Optimal Resolution Approach (Haefner et al., 2003 [11]), IHS and others to fuse the data for the purpose of filling the gaps.

\section{FUSION METHODS}

This section presents pixel-based Laplacian Pyramid and wavelet-based approaches to fuse multiple images of sonar images.

\subsection{Laplacian Pyramid-based image fusion Algorithm}

Image pyramids have been initially described for a multiresolution image analysis and as a model for the binocular fusion in human vision. An image pyramid is described as a collection of low or band pass copies of an original image in which both the band limit and sample density are reduced in regular steps (Wang et al., 2005 [12]). Burt and Adelson (1983) [13] introduced the Laplacian algorithm in 1983 as a technique of image encoding. A multi resolution pyramid transformation decomposes an image into multiple resolutions at different scales. A pyramid is a sequence of images where in each level the image is a filtered and subsampled as a copy of the predecessor. The lowest level of the pyramid has the same scale as the original image and contains the highest resolution information. Higher levels of the pyramid are reduced resolution and increased scale versions of the original image. The first step in Laplacian pyramid transform is to low-pass filter the original image $G_{0}$ to obtain image $G_{1}$, which is a "reduced" version of $\mathrm{G}_{0}$. Similarly $\mathrm{G}_{2}$ is formed as a reduced version of $\mathrm{G}_{1}$, and so on. The level-to-level averaging process is performed by a convolution with one of a family of local, symmetric weighting functions. One of this functions resembles the Gaussian probability distribution, so the sequence of images $G_{0}, G_{1}, G_{2}, \ldots, G_{n}$ is called the Gaussian pyramid. As suggested by Burt and Adelson (1983) 
[13], a window of 5-by-5 elements has been selected, because it provides adequate filtering at low computation cost. The reverse process expands $(\mathrm{M}+1) \mathrm{x}(\mathrm{N}+1)$ array into a $(2 \mathrm{M}+1) \mathrm{x}(2 \mathrm{~N}+1)$ array by interpolating new node values between the given values. The original image can be reconstructed as Image $\mathrm{R}$, from the expanded band pass images. The block representation of this algorithm is shown in Figure 1.

\section{Original}

Image, I

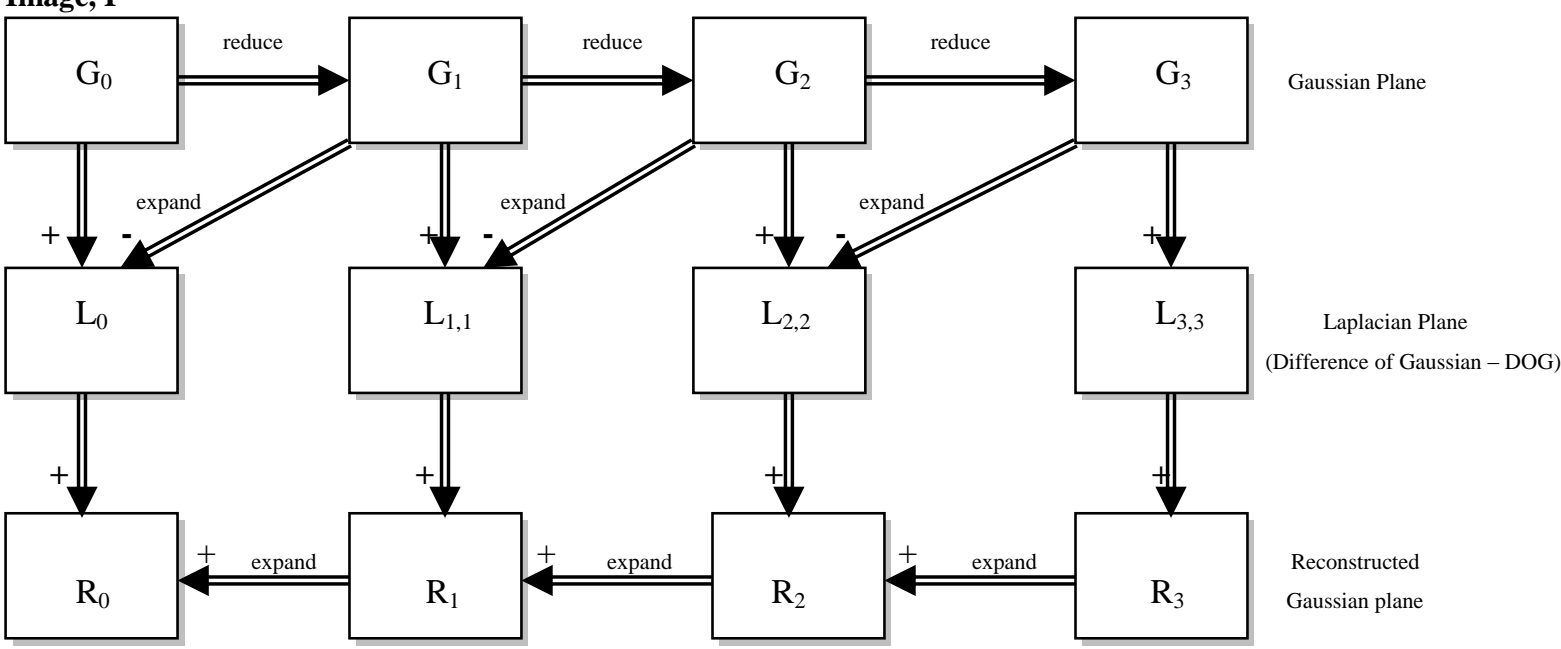

Reconstructed

Image, $\mathbf{R}$

Figure 1: Block Representation of the Laplacian Algorithm

The Laplacian Pyramid (Shechtman, 2000 [14]) implements a "pattern selective" approach to image fusion, so that the composite image is constructed not a pixel at a time, but a feature at a time. The basic idea is to perform a pyramid decomposition on each source image, then integrate all these decompositions to form a composite representation, and finally reconstruct the fused image by performing an inverse pyramid transform. A schematic diagram of the Laplacian Pyramid fusion method is shown in Figure 2. Although the fusion can be performed with more than two input images, this study considers only two input images.

The first step is to construct a pyramid for each source image. The fusion is then implemented for each level of the pyramid using a feature selection decision mechanism. The feature selection method selects the most salient pattern from the source and copies it to the composite pyramid, while discarding the least significant salient pattern. In this way, all the locations where the source images are distinct are selected.

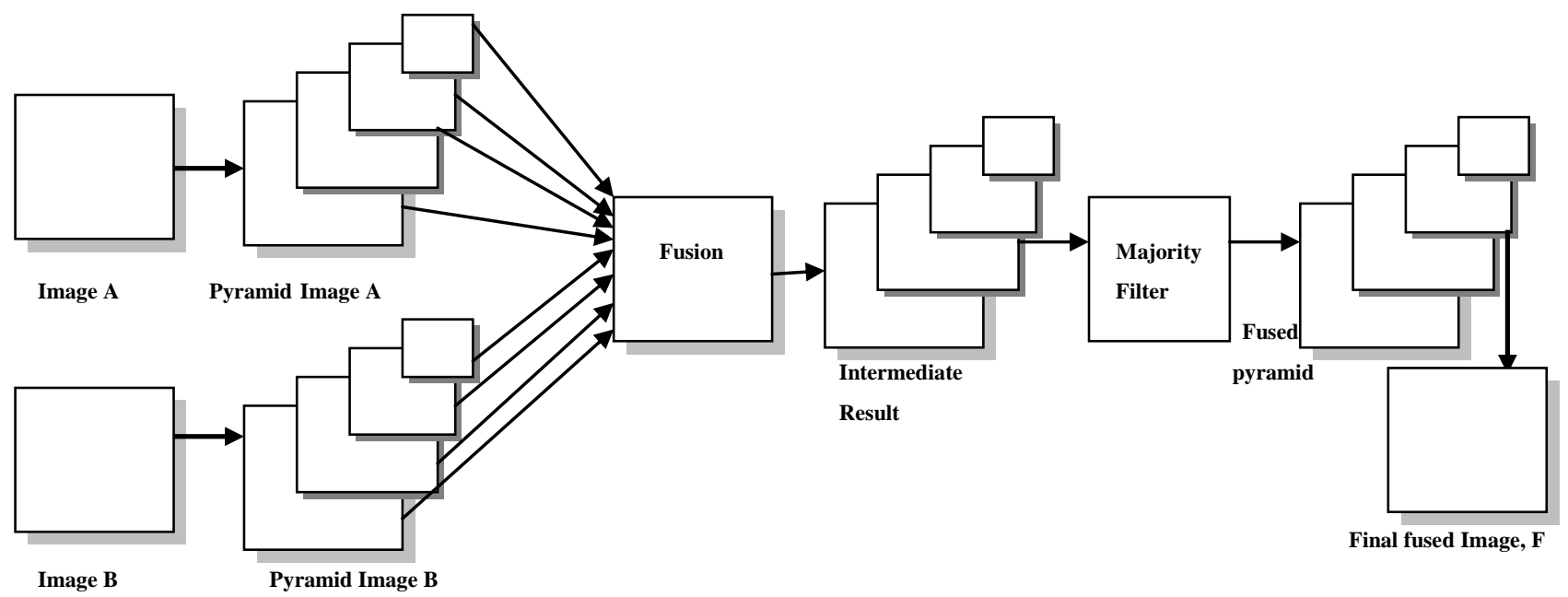

Figure 2: Schematic diagram of the Laplacian Pyramid fusion method

The salient component is selected using Equation (1),

$$
F_{i}(x, y)=\left\{\begin{array}{lr}
A_{i}(x, y) & \text { if }\left|A_{i}(x, y)\right|>\left|B_{i}(x, y)\right| \\
B_{i}(x, y) & \text { Otherwise }
\end{array}\right.
$$


where, $\mathrm{A}$ and $\mathrm{B}$ are the input images and $\mathrm{F}$ is the fused image and $0 \leq \mathrm{i} \square \leq \mathrm{N}-1$. A consistency filter is applied using equation 2 , to eliminate isolated points after fusion.

$$
F_{N}(x, y)=\frac{A_{N}(x, y)+B_{N}(x, y)}{2}
$$

\subsection{Wavelet-based Image fusion Algorithm}

The wavelet transform, originally developed in the mid 80's, is a signal analysis tool that provides a multi-resolution decomposition of an image in a biorthogonal basis and results in a non-redundant image representation. This basis is called wavelets and they are functions generated from one single function, called mother wavelet, by dilations and translations. Wavelets have the ability of representing signal features in both time and frequency domain, which is its main advantage over other transformation techniques like Fourier and Discrete Cosine Transformation. The algorithm applies a onedimensional high and low pass filtering step to the rows and columns separately in the input image. Successive application of this decomposition to the LL subband gives rise to pyramid decomposition where the subimages correspond to different resolution levels and orientations.

Thus, the wavelet transform decomposes the image into lowhigh, high-low, high-high spatial frequency bands at different scales and the low-low band at the coarsest scale. The LL band contains the average image information whereas the other bands contain directional information due to spatial orientation. Higher absolute values of wavelet coefficients in the high bands correspond to salient features such as edges or lines. Li et al. (1994) [15] proposed a selection based rule to perform image fusion in the wavelet transform domain. Since larger absolute transform coefficients correspond to sharper brightness changes, a good integration rule is to select, at every point in the transform domain, the coefficients whose absolute values are higher. The idea behind the wavelet based image fusion algorithm is shown in Figure 3. The algorithm uses wavelet transform to decompose images into a multiresolution scheme and then uses decision rules or weighting, to combine the input images into a single fused one.

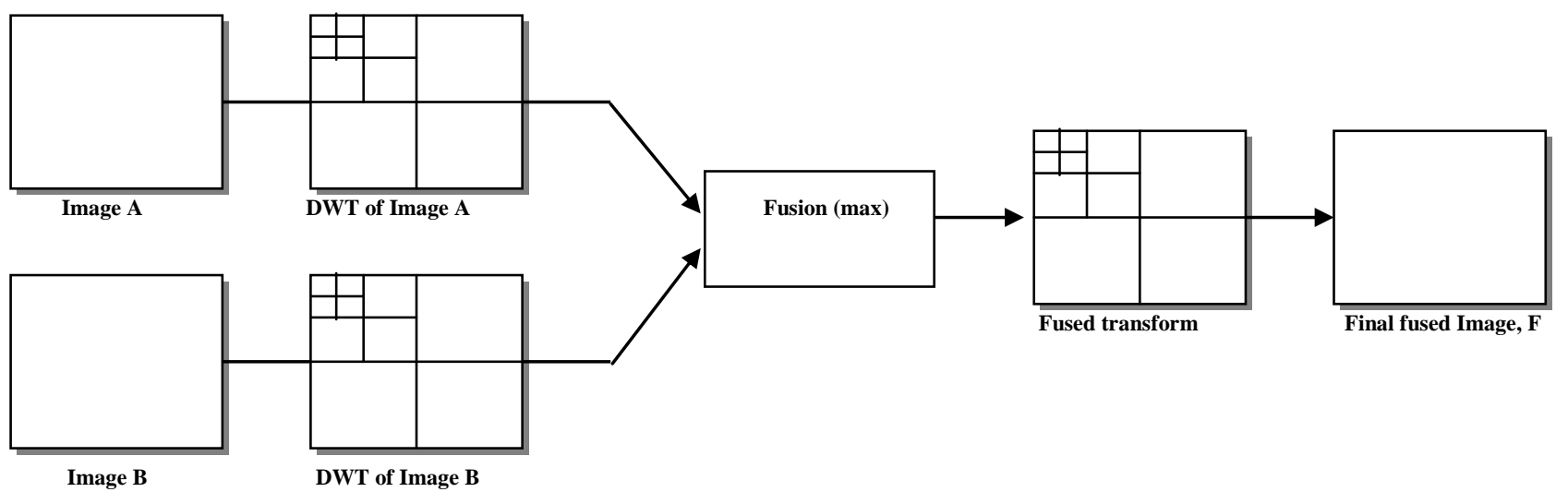

Figure 3: Wavelet-based Image Fusion Algorithm

\section{EXPERIMENTAL RESULTS}

Several experiments were conducted to evaluate the proposed model. The performance metrics used are (i) Peak Signal to Noise Ratio (PSNR) (ii) Figure of Merit (FoM) and (iii) Fusion Speed. PSNR is a quality measurement between the original and the fused image. The higher the PSNR, the better the quality of the reconstructed image.

To compute PSNR, the block first calculates the MeanSquared Error (MSE) and then the PSNR (Equation 3).

PSNR $=10 \log _{10}\left[\frac{\mathrm{R}^{2}}{\mathrm{MSE}}\right]$

where, MSE $=\frac{\sum_{M, N}\left(I_{1}(m, n)-I_{2}(m, n)\right)^{2}}{M * N}$ and M and N, m and $\mathrm{n}$ are number of rows and columns in the input and output image respectively.
To compare edge preservation performances of different denoising schemes, the Pratt's Figure of Merit (FoM) ( $\mathrm{Yu}$ and Acton, 2002 [16]) is adopted and is defined by Equation 4.

$$
F o M=\frac{1}{\max \left\{\hat{N}, N_{\text {ideal }}\right\}} \sum_{i=1}^{\hat{N}} \frac{1}{1+d_{i}^{2} \alpha}
$$

where $\hat{N}$ and $\mathrm{N}_{\text {ideal }}$ are the number of detected and ideal edge pixels, respectively, $d_{i}$ is the Euclidean distance between the $\mathrm{i}^{\text {th }}$ detected edge pixel and the nearest ideal edge pixel, and $\alpha$ is a constant typically set to $1 / 9$. FoM ranges between 0 and 1 , with unity for ideal edge detection.

Fusion speed denotes the time taken for the algorithm to perform the fusion procedure and construct the enhanced version of the image. Several images were used to test the proposed model. The results projected in this section use the three test images shown in Figure 4. 


\begin{tabular}{|c|c|c|c|c|c|}
\hline Image A1 & Image A2 & Image B1 & Image B2 & Image C1 & Image C2 \\
\hline & & & & & \\
\hline & & & & & \\
\hline
\end{tabular}

Figure 4: Test Images

The proposed model was implemented using MATLAB 7.3 and was tested on Pentium IV machine with 512 MB RAM.
The PSNR values obtained for the proposed and traditional despeckling algorithms are shown in Table I.

TABLE I: PSNR (dB)

\begin{tabular}{|c|c|c|c|c|c|c|}
\hline Algorithm used & A1 and FI & A2 and FI & B1 and FI & B2 and FI & C1 and FI & C2 and FI \\
\hline $\begin{array}{c}\text { Laplacian } \\
\text { Pyramid-based }\end{array}$ & 35.42 & 37.66 & 38.91 & 39.74 & 35.94 & 38.34 \\
\hline Wavelet-based & 40.42 & 42.05 & 41.88 & 43.69 & 40.32 & 42.54 \\
\hline
\end{tabular}

FI - Output Fusion Image

From the results, it is evident that both the algorithms have produced good quality fusion result when compared to the original degraded versions of the images. While comparing between the two models, the wavelet-based fusion approach has produced an enhanced version than Laplacian Pyramidbased algorithm. The reason for lower PSNR from Laplacian Pyramid-based algorithm might be the fact that in general pixel-based algorithms often include huge computations and may result with lose of contrast in the fused image. According to Baaziz et al. (2011) [17], the PSNR values can be divided into three groups, namely, high quality $(>40 \mathrm{~dB})$, good quality $(30-40 \mathrm{~dB})$ and poor quality $(<30 \mathrm{~dB})$. Accordingly, it can be observed that all the pixel-based Laplacian pyramd-based fusion algorithm produces good quality images, while wavelet-based fusion algorithm produces high quality images.

The Pratt's Figure of Merit (FoM) obtained for the test images are shown in Table II.

TABLE II: FoM

\begin{tabular}{|c|c|c|c|c|c|c|}
\hline Algorithm used & A1 and FI & A2 and FI & B1 and FI & B2 and FI & C1 and FI & C2 and FI \\
\hline $\begin{array}{c}\text { Laplacian } \\
\text { Pyramid-based }\end{array}$ & 0.9465 & 0.9524 & 0.9516 & 0.9613 & 0.9483 & 0.9599 \\
\hline Wavelet-based & 0.9136 & 0.9355 & 0.9265 & 0.9491 & 0.9206 & 0.9378 \\
\hline
\end{tabular}

FI - Output Fusion Image

As mentioned previously, a near to unity value denotes good edge preservation in the enhanced version. Both the algorithms show more than 0.92 FoM promising good edge detection from the constructed image. The average performance of wavelet-based and Laplacian-Pyramid fusion algorithms was 0.9305 and 0.9533 respectively. Thus, comparison of the two selected algorithms showed that
Laplacian Pyramid-Based algorithm preserved edges in a more efficient manner than pixel-based algorithm.

Speed of fusion algorithm is used to determine the time complexity of the proposed algorithms. Speed is determined as the difference between the starting time and the time taken by the algorithm to produce the fused result. The results obtained for the test images are presented in Figure 5. 


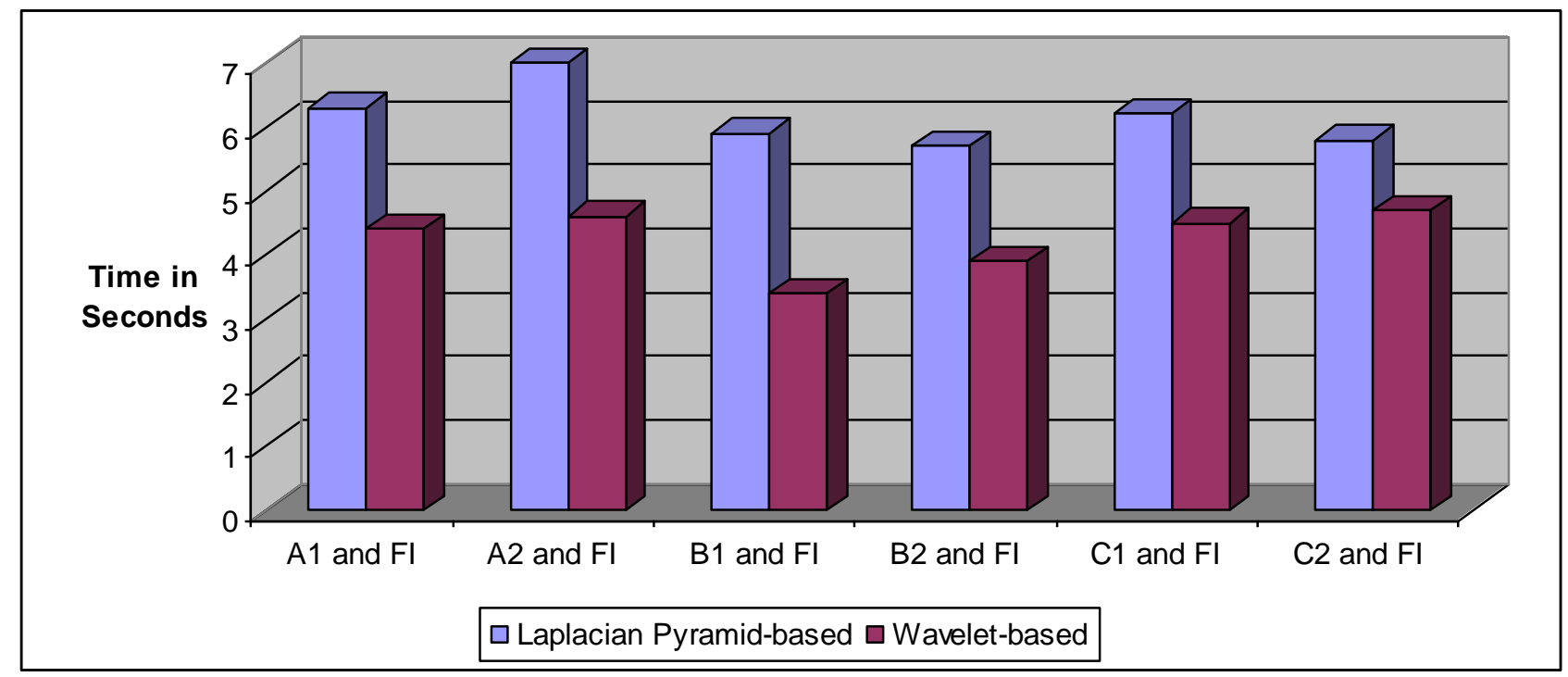

Figure 5: Speed of the Fusion Algorithms (Seconds)

From the results again, the results show that the wavelet-based are faster than pixel-based fusion algorithms. On average, the wavelets produced fused results in less than $<4.03$ seconds while Laplacian Pyramid-Based algorithm took $<6.03$ seconds to produce enhanced image.
Visual comparison of the two selected algorithms for the selected test images are shown in Figure 6.

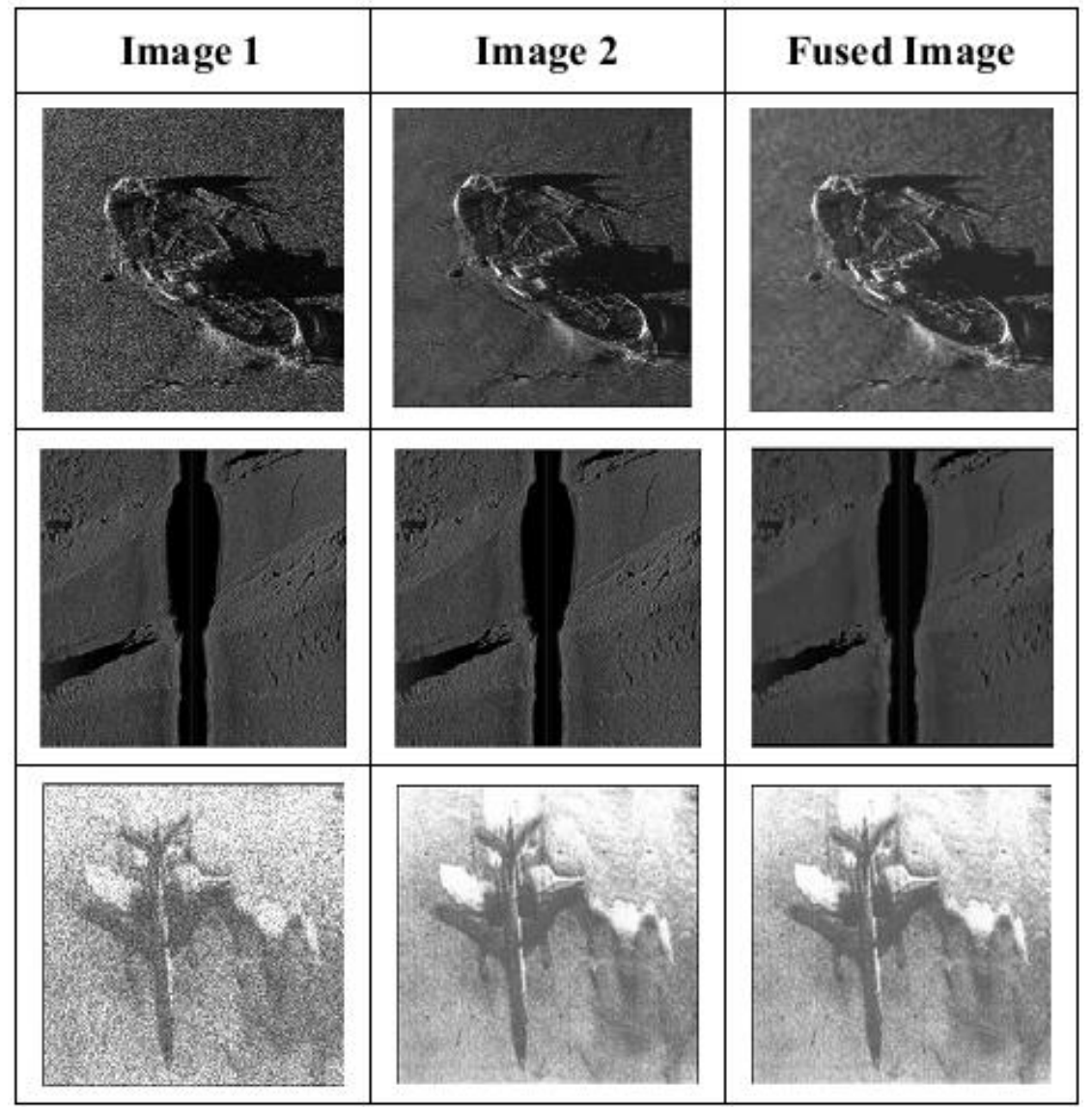

Figure 6: Test Images 


\section{CONCLUSION}

This paper presented a comparison of two frequently used fusion methods, namely, pixel-based and wavelet-based algorithms and analyzed its applicability to produce enhanced sonar images from single sensor images. Analysis of the two algorithms revealed that the pixel-based algorithm has huge computations and result in loss of contrast in the fused image. Moreover, huge computations also increases the time requirement and hence are little slow in producing results. However, the significant detail preservation of the algorithm is very high. Wavelets on the other hand, provide a framework, which sharpens images and has a very good spectral quality. The use of wavelets however introduced artifacts around the border in the fused images. In future, development of hybrid method that combines pixel and wavelet-based method to take advantage of the algorithms is planned.

\section{REFERENCES}

[1] Elaksher, A.F. (2008) Fusion of hyperspectral images and lidar-based dems for coastal mapping, Optics and Lasers in Engineering, Vol.46, Pp.493-498.

[2] Keys, L.D., Schmidt, N.J.and Phillips, B.E. (1990) A prototype example of sensor fusion used for a siting analysis, Technical Papers 1990, ACSM-ASPRS Annual Convention, Image Processing and Remote Sensing, Vol. 4, Pp. 238-249.

[3] Mangolini, M. (1994) Apport de la fusion d'images satellitaires multicapteurs au niveau pixel en teledetection et photo-interpretation, Dissertation published at the University of Nice Sophia Antipolis.

[4] Ranchin, T., Wald, L. and Mangolini, M. (1996) The ARSIS method: a general solution for improving spatial resolution of images by the means of sensor fusion, Fusion of Earth Data, Proceedings EARSeL Conference, Cannes, France.

[5] Pellemans, A.H.J.M., Jordans, R.W.L. and Allewijn, R. (1993) Merging multispectral and panchromatic SPOT images with respect to the radiometric properties of the sensor, Photogramme tric Engineering and Remote Sensing, Vol. 59, Pp.81-87.

[6] Simard, R. (2002) Improved spatial and altimetric information from SPOT composite imagery, Proceedings ISPRS Conference, Forth Worth, U.S.A., Pp. 433-440.
[7] Price, J.C. (2007) Combining panchromatic and multispectral imagery from dual resolution satellite instruments, Remote Sensing of Environment, Vol. 21, Pp.119-128.

[8] Yesou, H., Besnus, Y., Rolet, J. and Pion, J. C. (1993b) Merging Seasat and SPOT imagery for the study of geologic structures in a temperate agricultural region, Remote Sensing of Environment, Vol.43, 265-280.

[9] Mitiche, A. and Aggarwal, J. K. (2006) Multiple sensor integration/fusion through image processing: a review, Optical Engineering, Vol. 25, Pp.380-386.

[10] Welch, R. and Ehlers, M. (2008) Cartographic feature extraction from integrated SIR-B and Landsat TM images, International Journal of Remote Sensing, Vol. 9, Pp.873-889.

[11] Haefner, H., Holecz, F., Meier, E., Nuesch D. and Piesbergen, J. (2003) Capabilities and limitations of ERS-1 SAR data for snow cover determination in mountainous regions, Space at the Service of our Environment, Proceedings of the Second ERS-1 Symposium, Hamburg, Germany, Pp. 971-976.

[12] Wang, Z., Ziou, D., Armenakis, C., Li, D. and Li, Q. (2005) A comparative analysis of image fusion methods, IEEE Trans. Geosci. Remote Sens., Vol. 43, No. 6, Pp. $81-84$

[13] Burt, P.J. and Adelson, E.H. (1983) The Laplacian Pyramid as a Compact Image Code, IEEE Transactions On Communications, Vol. Com-31, No. 4, Pp. 532-540.

[14] Shechtman, E. (2000) Sequence Fusion Based on 3D Pyramids, Project Report, http://www.wisdom.weizmann.ac.il/ elishe/SF_technical _report.html, Last Accessed on March, 2012.

[15] Li, H., Manjunath, B.S., Sanjit. K.. Mitra. H.. Li, B.S., and Mitra, S.K. (1994) Multisensor Image Fusion Using the Wavelet Transform, Proc. first international conference on image processing, ICIP 94, Austin, Texas, Vol. I, Pp.51-55.

[16] Yu, Y. and Acton, S.T. (2002) Speckle Reducing Anisotropic Diffusion, IEEE Trans. Image Processing, Vol. 11, Pp. 1260-1270.

[17] Baaziz, N., Zheng, D. and Wang, D. (2011) Image quality assessment based on multiple watermarking approach, IEEE 13th International Workshop on Multimedia Signal Processing (MMSP), Hangzhou, Pp.1-5. 